ARTICLE

\title{
Progressive subcortical volume loss in treatment-resistant schizophrenia patients after commencing clozapine treatment
}

\author{
Giulia Tronchin ${ }^{1}$, Theophilus N. Akudjedu (D) ${ }^{1,2}$, Mohamed Ahmed ${ }^{1}$, Laurena Holleran ${ }^{1}$, Brian Hallahan ${ }^{1}$, Dara M. Cannon (D) and \\ Colm McDonald (iD)
}

The association of antipsychotic medication with abnormal brain morphometry in schizophrenia remains uncertain. This study investigated subcortical morphometric changes 6 months after switching treatment to clozapine in patients with treatment-resistant schizophrenia compared with healthy volunteers, and the relationships between longitudinal volume changes and clinical variables. In total, 1.5T MRI images were acquired at baseline before commencing clozapine and again after 6 months of treatment for 33 patients with treatment-resistant schizophrenia and 31 controls, and processed using the longitudinal pipeline of Freesurfer v.5.3.0. Two-way repeated MANCOVA was used to assess group differences in subcortical volumes over time and partial correlations to determine association with clinical variables. Whereas no significant subcortical volume differences were found between patients and controls at baseline $(F(8,52)=1.79 ; p=0.101)$, there was a significant interaction between time, group and structure $(F(7,143)=$ 52.54; $p<0.001)$. Corrected post-hoc analyses demonstrated that patients had significant enlargement of lateral ventricles $(F(1,59)=$ $48.89 ; p<0.001)$ and reduction of thalamus $(F(1,59)=34.85 ; p<0.001)$, caudate $(F(1,59)=59.35 ; p<0.001)$, putamen $(F(1,59)=87.20$; $p<0.001)$ and hippocampus $(F(1,59)=14.49 ; p<0.001)$ volumes. Thalamus and putamen volume reduction was associated with improvement in PANSS $(r=0.42 ; p=0.021, r=0.39 ; p=0.033)$, SANS $(r=0.36 ; p=0.049, r=0.40 ; p=0.027)$ and GAF $(r=-0.39$; $p=0.038, r=-0.42 ; p=0.024)$ scores. Reduced thalamic volume over time was associated with increased serum clozapine level at follow-up ( $r=-0.44 ; p=0.010)$. Patients with treatment-resistant schizophrenia display progressive subcortical volume deficits after switching to clozapine despite experiencing symptomatic improvement. Thalamo-striatal progressive volumetric deficit associated with symptomatic improvement after clozapine exposure may reflect an adaptive response related to improved outcome rather than a harmful process.

Neuropsychopharmacology (2020) 45:1353-1361; https://doi.org/10.1038/s41386-020-0665-4

\section{INTRODUCTION}

Approximately $30 \%$ of patients with schizophrenia meet criteria to be considered treatment resistant [1, 2], usually defined as the failure to respond to at least two adequate trials of antipsychotic medication [3]. Clozapine has an established superior clinical effect to control symptoms in treatment-resistant patients, with $60-70 \%$ having a positive response $[4,5]$. Patients treated with clozapine also often experience troublesome side effects, including the significant weight gain and lipid abnormalities [6], which notably have been associated with improvement in symptomatology $[7,8]$. Cross-sectional MRI studies of patients with treatment-resistant schizophrenia (TRS) receiving clozapine and other antipsychotic medications have reported a range of brain abnormalities compared with controls, including reduced global grey matter $[9,10]$, predominantly in frontal and temporal regions [11-13] and volumetric reduction of the amygdala and hippocampus $[12,13]$.

The association of antipsychotic medication use with progressive brain deficits has been explored in longitudinal studies of schizophrenia [14, 15]. These studies mostly use an observational rather than randomised design approach and thus cannot fully account for illness or service-related factors, which influence clinician and patient medication choice. In a meta-analysis of longitudinal MRI studies based on 1155 patients with schizophrenia and 911 healthy controls, Vita et al. [15] reported reduced cortical grey matter volume over time in patients, which was related to cumulative exposure and mean daily dose of antipsychotic medications. Patients treated with first-generation antipsychotic (FGA) medications compared with secondgeneration antipsychotics (SGA) displayed more progressive grey matter loss, which correlated with higher mean daily antipsychotic dose. Likewise, van Haren et al. [16] 5-year longitudinal study reported an association between higher cumulative dose of FGA over time and more marked cortical thinning, while higher dose of SGA in contrast was associated with less cortical thinning. However, patients who received clozapine treatment during the interscan interval showed more pronounced superior temporal cortical thinning compared with those not treated with clozapine. In contrast, in another analysis of this cohort, higher cumulative dose of clozapine during the interscan interval was related to the attenuated loss of grey matter in the left superior frontal gyrus [17].

\footnotetext{
${ }^{1}$ Centre for Neuroimaging \& Cognitive Genomics (NICOG), Clinical Neuroimaging Laboratory, NCBES Galway Neuroscience Centre, College of Medicine Nursing and Health Sciences, National University of Ireland Galway, Galway H91TK33, Ireland and ${ }^{2}$ Institute of Medical Imaging \& Visualisation, Faculty of Health \& Social Science, Department of Medical Science \& Public Science, Bournemouth University, Bournemouth, UK

Correspondence: Giulia Tronchin (giulia.tronchin@nuigalway.ie)
}

Received: 8 January 2020 Revised: 20 March 2020 Accepted: 24 March 2020

Published online: 8 April 2020 
Table 1. Characteristics of patients with treatment-resistant schizophrenia and controls.

\begin{tabular}{llll}
\hline & $\begin{array}{l}\text { Patient group } \\
(n=33)\end{array}$ & $\begin{array}{l}\text { Control group } \\
(n=31)\end{array}$ & Test statistic/p value \\
\hline Sex $(\mathrm{m} / \mathrm{f})$ & $23 / 10$ & $20 / 11$ & $X^{2}=0.19 ; 0.660$ \\
Age at onset (years) & $22.8 \pm 0.8$ & $39.3 \pm 10.6$ & $t=1.10 ; 0.274$ \\
Age at baseline (years) & $36.4 \pm 10.7$ & $(23-59)$ & $t .4 \pm 3.2$ \\
Age range & $(22-61)$ & & $t=1.21 ; 0.230$ \\
Time between baseline and follow-up MRI scans (months) & $6.6 \pm 1.7$ & $1,591,515.15 \pm 27,500.42$ & $t=0.46 ; 0.644$ \\
IIIness duration before commencing clozapine (years) & $13.6 \pm 8.8$ & $1,610,322.58 \pm 29,886.83$ & \\
Intracranial volume $\left(\mathrm{mm}^{3}\right)$ & &
\end{tabular}

Longitudinal subcortical neuroimaging studies specifically of treatment-resistant clozapine-naïve patients are sparse, with small numbers of participants or without a matched control group. An early study of subcortical structures by Chakos et al. [18] based on 15 patients, and without a control group, reported a $10 \%$ decrease in caudate volume after 55 weeks, when switched from treatment with typical antipsychotic medications to clozapine. In contrast, patients who stayed on typical antipsychotic medications displayed an $8 \%$ enlargement in the caudate. In another study of 26 patients by Scheepers et al. [19] volume reduction of caudate nucleus was identified after 24 weeks of treatment with clozapine. There was no neuroanatomical correlation with clinical response. In the same cohort, after 52 weeks of treatment, reduced volume of the left caudate was greater in patients who responded to treatment compared with non-responders [20]. Another small study with eight patients and eight controls reported reduced caudate volume after 2 years of treatment with clozapine, with analogous results for the putamen, which was not statistically significant [21]. Thus, these early studies consistently indicate that switching patients from FGA medication to clozapine is associated with a decrease of caudate volume over time, and has generally been interpreted as a correction by clozapine of caudate hypertrophy induced by FGA medication due to their potent dopamine blockade and the high concentration of dopamine receptors in the caudate [22]. However, nowadays most patients are already taking SGA medications prior to clozapine commencement and it remains unclear whether switching to clozapine in such circumstances would have a similar effect on the basal ganglia. Furthermore, other subcortical structures such as the hippocampus and thalamus have not been investigated in longitudinal studies of switching to clozapine.

Given the importance of identifying factors predicting response to clozapine, the association of clinical response with baseline alterations in subcortical structures has also been studied, with conflicting results. In a randomised controlled trial by Arango et al. [23], whereas larger right prefrontal cortex predicted improvement in SANS scores compared with haloperidol treated patients, there was no such association between clinical symptom change and caudate or hippocampal volume at baseline. Smaller hippocampal volume compared with healthy controls at baseline predicted improvement in disorganised symptoms over time in a longitudinal study by Molina et al. [24]. In another longitudinal study, decreased left caudate volume over time was related to a significant improvements in positive and general symptoms, but not negative symptoms [20].

We have previously investigated cortical anatomy in a sample of patients before and after switching to clozapine in comparison to healthy volunteers [25], and demonstrated on-going cortical thinning in TRS patients over a 6-month period, in particular for younger patients. The present study, using a unique sample of treatment-resistant clozapine-naïve schizophrenia patients, offers a novel opportunity to comprehensively investigate whether subcortical structures demonstrate progressive neuroanatomical changes after 6 months of clozapine treatment and whether any such changes are related to clinical variables, including treatment response and amount of clozapine taken.

\section{METHOD}

Participants

As previously reported [25] 39 patients with TRS prior to clozapine initiation and 40 healthy volunteers $(\mathrm{HC})$ were initially recruited for the baseline assessment. At the follow-up, 33 patients, after 6 months of treatment with clozapine and a total of 31 healthy controls, matched for sex and age, were successfully re-recruited, scanned and assessed (Table 1). Patients were included if aged 18-60 years and clinically due to switch to clozapine because of treatment resistance. Patients and controls were excluded from the study if they had a previous trial of clozapine treatment, a learning disability, history of neurological illness, history of head injury, which resulted in the loss of consciousness for over $5 \mathrm{~min}$, treatment with oral steroid in the 3 months prior to participation, history of comorbid alcohol/substance dependency as defined by the DSM-IV criteria or any contraindication to MRI scanning. Exclusion criteria for controls also included a current or past axis I mental disorder or any psychotic disorder in a first-degree relative. The study was approved by the Clinical Research Ethics Committee, Galway University Hospitals. Fully informed written consent was obtained for all participants.

Clinical assessment

All patients were diagnosed using the Diagnostic and Statistical Manual for Mental Disorders Fourth Edition Text Revision (DSM-IVTR) (American Psychiatric Association, 2000). Treatment resistance was defined as the failure to respond to at least two adequate trials of antipsychotic medications, including at least one atypical antipsychotic drug, with a prolonged period of moderate to severe positive and/or negative symptoms [26]. The severity of positive and negative symptoms was assessed at both time points using the Positive and Negative Syndrome Scale (PANSS) [27], the Scale for the Assessment of Positive Symptoms (SAPS) [28] and the Scale for the Assessment of Negative Symptoms (SANS) [29]. Social, occupational and psychological functioning was assessed using a Global Assessment of Functioning Score [30]. We used the symptomatic remission criteria of Andreasen et al. [31] with the exclusion of the maintenance over 6-month observation period [32]. Remission at the 6-month follow-up assessment was therefore defined as having scores of mild or less (item scores of $\leq 2$ using the 0-6 range) on all eight of the following PANSS items: delusions (P1), conceptual disorganisation (P2), hallucinatory behaviour (P3), blunted affect (N1), social withdrawal (N4), lack of spontaneity (N6), mannerisms/posturing (G5) and unusual thought content (G9). 
Table 2. Clinical features of patient group at baseline and follow-up $(n=33)$.

\begin{tabular}{|c|c|c|c|}
\hline & $\begin{array}{l}\text { Baseline } \\
(\text { mean } \pm S D)\end{array}$ & $\begin{array}{l}\text { Follow-up } \\
\text { (mean } \pm \text { SD) }\end{array}$ & Test statistic/p value \\
\hline \multicolumn{4}{|l|}{ Clinical scales } \\
\hline PANSS negative score & $16.2 \pm 7.0$ & $9.1 \pm 7.1$ & $z=-4.51^{\mathrm{a}} ;<0.001$ \\
\hline PANSS general score & $24.1 \pm 8.9$ & $11.7 \pm 8.3$ & $z=-4.90^{\mathrm{a}} ;<0.001$ \\
\hline PANSS total score & $54.3 \pm 17.8$ & $26.9 \pm 17.6$ & $t=10.04 ;<0.001$ \\
\hline SAPS & $28.0 \pm 16.3$ & $13.2 \pm 11.0$ & $z=-4.45^{\mathrm{a}} ;<0.001$ \\
\hline Global assessment of functioning & $46.8 \pm 10.8$ & $64.9 \pm 14.1$ & $t=13.12 ;<0.001$ \\
\hline \multicolumn{4}{|l|}{ Medications } \\
\hline Typical antipsychotics $(n)$ & 5 & 0 & \\
\hline Atypical antipsychotics $(n)$ & 33 & 2 & \\
\hline \multicolumn{4}{|l|}{ Anthropomorphic measurements } \\
\hline Weight $(\mathrm{kg})$ & $85.9 \pm 15.4$ & $90.1 \pm 16.6$ & $t=-3.31 ; 0.002$ \\
\hline Waist circumference $(\mathrm{cm})$ & $97.8 \pm 12.1$ & $103.1 \pm 13.4$ & $t=-4.94 ;<0.001$ \\
\hline Body Mass Index & $28.0 \pm 4.9$ & $29.3 \pm 5.0$ & $z=-2.78^{\mathrm{a}} ; 0.005$ \\
\hline Total cholesterol (mmol/L) & $4.8 \pm 1.1$ & $5.5 \pm 0.8$ & $t=-3.38 ; 0.003$ \\
\hline Triglycerides (mmol/L) & $1.8 \pm 1.0$ & $2.5 \pm 1.4$ & $z=-2.62^{\mathrm{a}} ; 0.009$ \\
\hline
\end{tabular}

MRI data acquisition

MRI images were acquired for all participants at baseline and after 6 months at University Hospital Galway in a 1.5 Tesla Siemens Magnetom Symphony scanner (Erlangen, Germany) equipped with a four-channel head coil. A magnetisation prepared rapid gradient echo sequence was acquired to generate high resolution volumetric T1-weighted images, with the following parameters: repetition time: $1140 \mathrm{~ms}$, echo time: $4.38 \mathrm{~ms}$, inversion time: 600 $\mathrm{ms}$, flip angle: $15^{\circ}$, matrix size: $256 \times 256$, interpolated to $512 \times$ 512, slice thickness: $0.9 \mathrm{~mm}$ and in-pixel resolution: $0.45 \mathrm{~mm}^{2}$.

\section{MRI processing}

Volumetric T1-weighted images used in the analyses were intensity inhomogeneity corrected using non-parametric, nonuniform intensity normalisation (N3) [33] as previously reported $[25,34]$. Eight subcortical regions-of-interest (ROI): lateral ventricle, thalamus, hippocampus, caudate, putamen, globus pallidus, amygdala and nucleus accumbens were bilaterally segmented using the longitudinal pipeline of Freesurfer v.5.3.0 [35, 36]. Specifically, this technique is based on an unbiased within-subject anatomical template [35], created using a robust and inverse consistent registration method [37], is able to overcome the limitations of longitudinal processing methods. It reduces the risk of underestimating change, giving an unbiased estimation of the neuroanatomical structure volume over time, removing asymmetry-induced processing bias and avoiding overregularisation or temporal smoothness constraints [35]. This technique has also sufficient sensitivity and reliability for small sample sizes [35]. The several steps of the processing pipeline to obtain the output have previously been described in detail [38]. Intracranial volume (ICV) is computed by dividing a predetermined constant with the factor by which the input magnetic resonance (MR) images are scaled in size to align to the MNI305 head atlas [39-41]. At each time point, quality check of the segmentation output was performed, which involves a visual inspection at each of the analysis stages, to verify that the segmentation was anatomically accurate and computationally successful [42]. Six images failed the quality check and required manual editing using control points to fix intensity normalisation [43]. Following quality check and manual editing, no images were excluded. Subsequently subcortical volumes were bilaterally extracted and summed together to obtain one measure for each ROI.

Statistical analysis

Statistical Package for the Social Sciences version (SPSS Inc., v23, IBM, New York, USA) was used to carry out all analyses. The Shapiro-Wilks test was used to test for normal distribution of demographics, clinical, neuroanatomical and anthropomorphic variables, with outliers defined as greater or less than three standard deviations from the mean. Age, gender and time between scanning were compared between groups using either a $t$-test, Chi-square or Mann-Whitney $U$ Test. Differences between baseline and follow-up on clinical variables and anthropomorphic measurements were tested using the Wilcoxon Signed Ranks and Paired-Sample $t$-test. An initial one-way multivariate analysis of covariance (MANCOVA) was performed to evaluate differences between groups at baseline on the eight subcortical structures, covarying for age, sex and ICV. Post-hoc analyses were performed to assess differences at baseline on the eight subcortical structures between controls and patients previously treated with atypical and/or typical medications. Thereafter two-way repeated MANCOVA was used to assess the course of changes in volume of subcortical structures over time between groups, covarying for age, sex and ICV. The group-by-age interaction was used to 


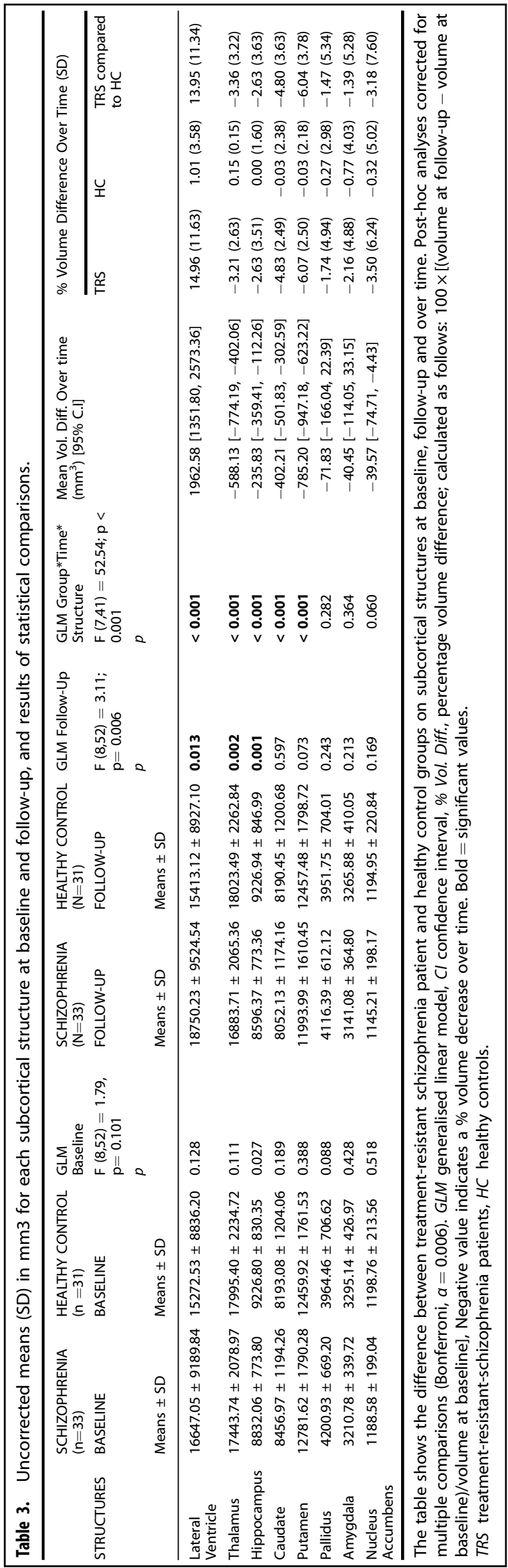

determine the effect of age on anatomical change between groups over time. Post-hoc analysis, corrected for multiple comparison (Bonferroni, $a=0.006$ ) was carried out to clarify which regions were significantly changing over time. An additional one-way MANCOVA and subsequently a two-way repeated MANCOVA was performed to assess differences between clozapine responders and non-responders at baseline and over time on subcortical structures, covarying for age, sex and ICV. Partial correlations were carried out controlling for the potential influence of age, sex and ICV on the relationship between the subcortical brain regions, which showed a significant change over time $\left(\frac{\text { follow-up-baseline }}{\text { baseline }} \times 100\right)$ and change in PANSS, SANS, SAPS and GAF (follow-up-baseline) [10]. These correlations were hypothesis driven and not corrected for multiple comparisons. Pearson correlation analyses were performed to explore the relationship between subcortical structures showing a significant change over time in TRS patients and the variables age, duration of illness, body mass index (BMI), daily dose and serum level of clozapine at follow-up.

\section{RESULTS}

Clinical characteristics

Patient and control groups did not differ across age, sex and time between scans (Table 1). Patients after treatment with clozapine displayed a substantial and statistically significant improvement in each symptom and function rating scale. At follow-up, patients also displayed a significant increase of weight, waist, BMI, total cholesterol and triglycerides compared with baseline (Table 2). Twelve patients had previously been prescribed typical antipsychotic drugs and five were still taking FGA medications at the point of the baseline scan. At baseline before switching to clozapine, 21 patients were on monotherapy with one SGA medication (olanzapine $=7$, quetiapine $=4$, aripiprazole $=4$, amisulpride $=1$, paliperidone $=1$, risperidone long acting injection $=1$ ), 10 patients were treated with two antipsychotic medications (olanzapine + another antipsychotic $=7$ ), with 1 patient treated with three and another patient treated with four antipsychotic medications. At follow-up 16 patients (48\%) were in remission.

Differences between groups on subcortical regions at baseline and over time

There was no significant difference between TRS patients and controls at baseline ( $n=33$ TRS; $n=31 \mathrm{HC}$ ) when considering jointly the eight subcortical structures and taking account of multiple comparisons $(F(8,52)=1.79 ; p=0.101$, Table 3$)$. We also assessed for differences in subcortical structures at baseline in the larger initially recruited sample ( $n=39$ TRS; $n=40 \mathrm{HC}$ ). Volumetric changes in structures such as hippocampus and lateral ventricles did not survive overall multiple comparison correction $(F(8,66)=$ $1.82 ; p=0.088$, Supplementary Table 1 ), but were in keeping with the effects sizes (circa 0.5) identified for such structures in larger case control samples of patients with schizophrenia [44]. However, a strong significant overall interaction between time, group and brain structure was demonstrated $(F(7,143)=52.54 ; p<0.001$, Table 3). Post-hoc analyses, robustly corrected for multiple comparison (Bonferroni, $a=0.006$ ), revealed a significant volumetric increase in lateral ventricle $(F(1,59)=48.89 ; p<0.001$, Fig. 1a) and decrease in thalamus $(F(1,59)=34.85 ; p<0.001$, Fig. $1 \mathrm{~b})$, caudate $(F(1,59)=59.35 ; p<0.001$, Fig. $1 \mathrm{c})$, putamen $(F(1,59)=87.20 ; p<0.001$, Fig. $1 d)$ and hippocampus $(F(1,59)=$ 14.49; $p<0.001$, Fig. 1e) volumes for patients compared with healthy controls (Table 3 ). The relative consistency of the progressive volumetric changes in the patient cohort is apparent from the individual level data points displayed in Supplementary Fig. 1. There was no significant group-by-age interaction on the 
A

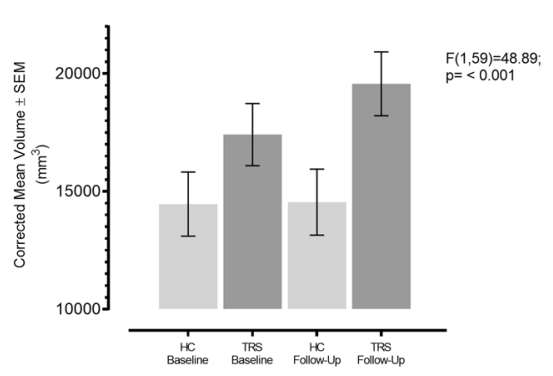

D

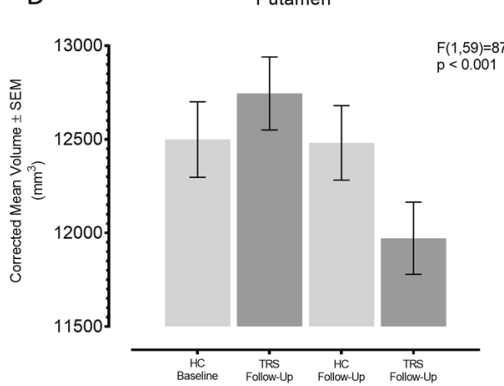

B

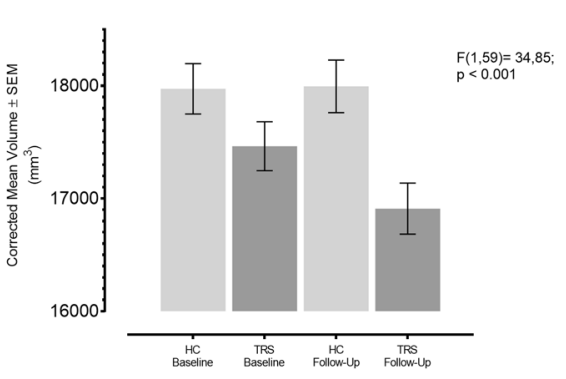

E

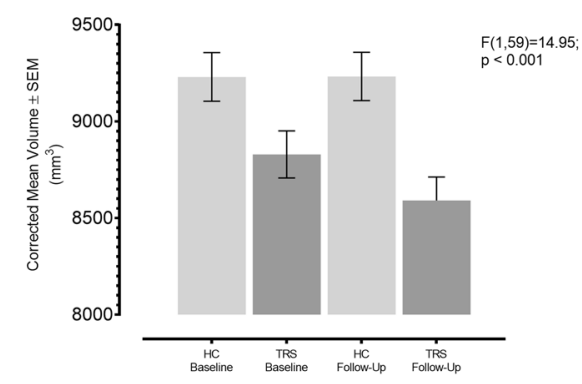

C

Caudate

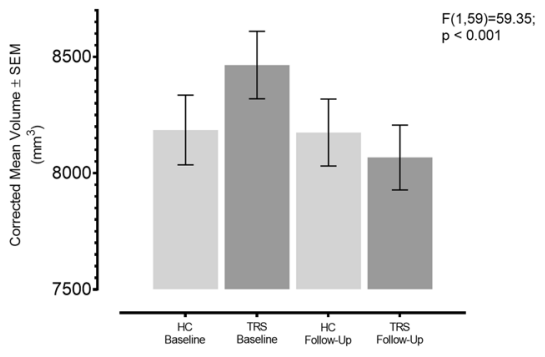

Fig. 1 a, b, c, d, e. Volumetric change over time on subcortical structures. Plots of subcortical structures that presented significant changes over time in treatment-resistant schizophrenia patients compared with healthy controls. Note: all values corrected for age, sex and ICV.

A
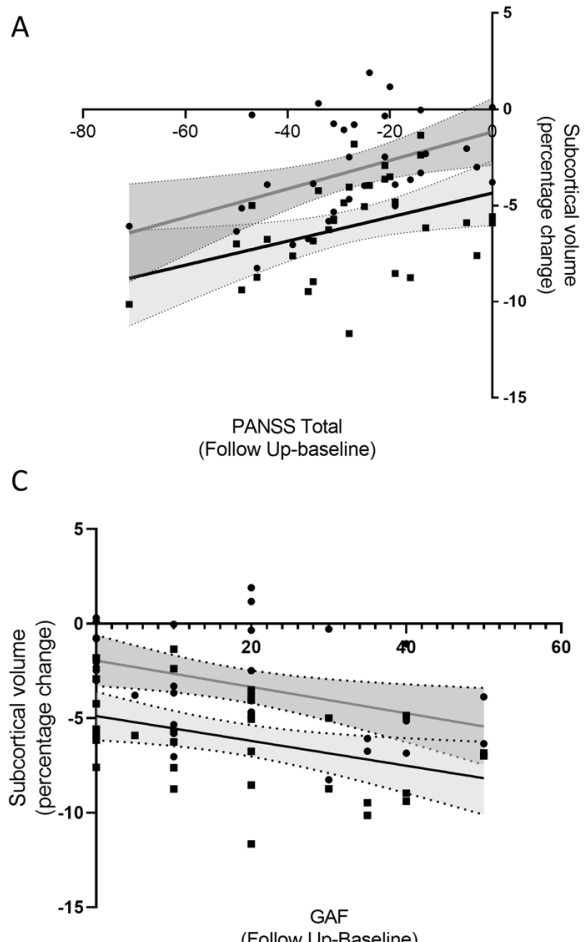

B

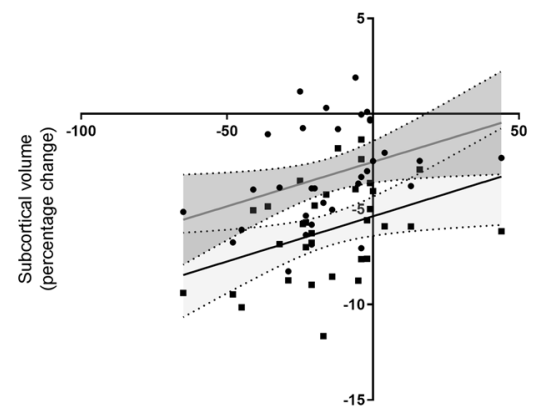

- Thalamus

$r=0.36 ; p=0.049$

Putamen

$r=0.40 ; p=0.027$

$95 \% \mathrm{Cl}$

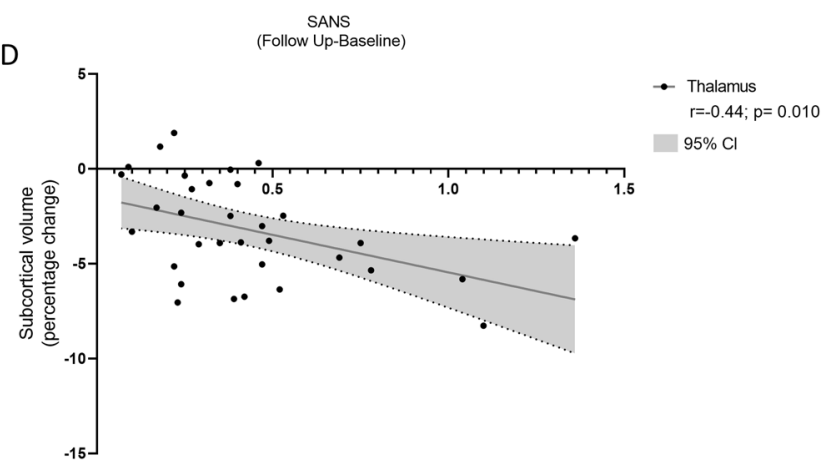

Serum levels of clozapine at Follow Up
$(\mathrm{ng} / \mathrm{ml})$

$r=-0.39 ; p=0.043$

- Putamen

$r=-0.42 ; p=0.028$

$95 \% \mathrm{Cl}$

Fig. 2 Correlations between neuroanatomy and clinical variables. Association between percentage of volume change in thalamus and putamen and change in (a) PANSS total score (b) SANS (c) GAF and (d) association between percentage of volume change in thalamus and level of serum of clozapine at follow-up.

progression of the subcortical structures between patients and controls $\quad(F(84,112)=1.13 ; \quad p=0.272)$. Post-hoc analysis revealed no significant differences at baseline between controls and patients previously treated with atypical and/or typical medications when considering the eight subcortical structures $(F(8,16)=1.49 ; p=0.117)$.
Response to clozapine and subcortical changes at baseline and over time

When investigating the baseline differences between those who remitted on clozapine treatment $(n=16)$ and non-responders $(n=17)$ for the eight subcortical structures, no significant differences were revealed $(F(8,21)=1.32 ; p=0.286)$. Likewise, 
there was no significant overall effect of time on subcortical brain structures between patients responding to clozapine compared with patients non-responders $(F(7,20)=0.50 ; p=0.834)$.

Correlation between neuroanatomy and clinical variables in treatment-resistant patients

In TRS patients, when covarying for age, sex and ICV, volumetric reduction of thalamus and putamen over time were significantly associated with improvement in PANSS Total score $(r=0.42, p=$ $0.021 ; r=0.39, p=0.033$, respectively, Fig. 2a) and improvement in negative symptoms assessed with the SANS scale $(r=0.36, p=$ $0.049 ; r=0.40, p=0.027$, respectively, Fig. 2b). Similarly, improvement in PANSS general score was significantly related to decreased volume in thalamus over time $(r=0.39 ; p=0.034)$. Controlling for serum clozapine level at follow-up and duration of illness did not impact on the above findings, however, improvement of GAF was additionally found to relate to reduced thalamic $(r=-0.39 ; \quad p=0.038)$ and putaminal $(r=-0.42 ; \quad p=0.024)$ volume (Fig. $2 \mathrm{c}$ ). Improvement in SAPS was associated with reduced putaminal volume $(r=0.39 ; p=0.035)$, but this association weakened slightly and lost significance $(r=0.31 ; p=0.102)$ when removing one outlier who demonstrated a $76 \%$ improvement in positive symptoms. No other associations were found between change in other subcortical brain structures and clinical variables (Supplementary Table 2).

Exploratory analyses between structures showing significant change over time in patients and treatment-related factors When exploring the association between changes over time in subcortical structures and treatment-related factors in patients, including BMI change, and serum clozapine at follow-up, a significant association was identified between reduced volume of the thalamus over time and increased clozapine serum level at follow-up ( $r=-0.44 ; p=0.010$, Fig. $2 d$ ), with this correlation strengthening $(r=-0.49 ; p=0.010)$ when controlling for change in clinical symptoms (PANSS, SAPS, SANS) and functioning (GAF).

\section{DISCUSSION}

To the best of our knowledge, this is the largest sample to date to examine the effects of switching to clozapine on subcortical regions in a relatively clinically homogenous sample of TRS patients using a longitudinal semi-automated subject-specific approach (Freesurfer v.5.3.0) [36]. In this longitudinal study, minor subcortical differences were detected between patients and controls at baseline, which failed to survive multiple comparisons correction. However, we identified substantial progressive volumetric reduction of the thalamus, hippocampus, caudate, putamen and enlargement of lateral ventricles over a 6-month period in patients compared with controls. Reduced caudate volume over time has been consistently reported in the majority of studies of patients switched from typical antipsychotic medications to clozapine [18-21] and has been interpreted as reversal of previous enlargement due to excessive dopamine blockade. Consistent with this, longitudinal studies demonstrate basal ganglia enlargement when taking typical medications was reversed by switching to atypical antipsychotic medications [45, 46]. Reduction of thalamic volume over time was also reported in a 5-year longitudinal voxel-based morphometry study [17]. However, no association has previously been reported between cumulative doses of clozapine and subcortical deficits. The hippocampal progressive reduction identified in this cohort on switching to clozapine has not been previously reported, but notably the direction of change is in keeping with the other subcortical structures. The lateral ventricle enlargement over time could be interpreted as ventricular expansion as a result of the significant reduction of surrounding subcortical regions [47]. The degree of volumetric change in this cohort after 6 months in regions such as the hippocampus and lateral ventricles is comparable to the rate of change detected in previous longitudinal studies over longer time periods $[48,49]$. The high density of dopamine D2 receptors [50] in basal ganglia and other structures, such as thalamus and hippocampus, renders them major targets to which dopaminergic pathways project [51]. In a preclinical study, Guma et al. [52], presented evidence that D2 receptors play a significant role in mediating antipsychotic induced structural changes, whereby volumetric reduction in cortical areas, hippocampus and thalamus, was induced by genetic deletion of D2 receptors.

Our study did not detect any difference in subcortical structures between those who achieved clinical remission with clozapine treatment and non-responders, either at baseline or over time, consistent with some previous studies $[9,19]$. In one longitudinal study of patients (which did not include a control sample), responders showed a significant reduction in left caudate volume after 24 weeks of clozapine treatment [20].

These results lead us to speculate on three reasons for the lack of significant baseline subcortical volume deficits in patients compared with controls in this cohort and the subsequent marked progressive volume loss over time after commencing clozapine. (i) Direct effects of clozapine treatment, (ii) withdrawal of prior treatment with other medications or (iii) illness progression independent of medication use.

(i) This cohort of TRS patients may be a categorically different illness subtype with different underlying mechanisms and pathophysiology compared with D2 receptor antagonist responsive schizophrenia [53, 54]. Lack of the striatal dopaminergic elevation in TRS, typical in schizophrenia could explain why treatment with dopamine antagonists is ineffective as they target the wrong processes [55]. Abnormal glutamatergic function, with higher glutamate + glutamine level concentrations, has been reported in TRS compared with first-line responders [54, 56]. Indeed it has been suggested that clozapine's efficacy might relate to its ability to attenuate glutamate release, as demonstrated in preclinical studies [57]. In our cohort the previous lack of symptomatic response to typical and atypical antipsychotic medications may have related to relative subcortical volume preservation compared with healthy controls. Hence, the subcortical volume loss after commencing clozapine treatment may directly have been related to clozapine efficacy [19]. Indeed, crosssectional studies on neuroanatomy of TRS patients are usually on patients already receiving clozapine, and demonstrate reduction of cortical and subcortical volumes $[9,12,13]$, as we see at the follow-up point in our study when patients are on clozapine treatment. It may also be that acutely symptomatic phase of illness is linked to increased neuroinflammation, which has been associated with increases in local blood flow, vascular permeability, microglia activation and extracellular volume [58]. In this scenario, successful treatment with clozapine might have resulted in an anti-inflammatory process [59] that reversed these inflammatory changes, resulting in subcortical volume reduction.

(ii) Prior exposure of this cohort to antipsychotic medications over the years might have ameliorated or corrected diseaserelated volume loss $[15,16,44,60]$, which may explain our finding of only minor baseline volume differences. Interestingly unmedicated patients have been reported to display greater subcortical deficits, especially of the caudate and thalamus, compared with medicated patients $[44,61]$. On this interpretation, the progressive brain volume change of subcortical structures on switching to clozapine treatment might have been related to the withdrawal of other atypical antipsychotic medications. The neurobiological mechanism that underlies the progressive volumetric loss of subcortical structures is still unknown, however, neural apoptosis, necrosis, synaptic pruning might play a role in producing volume deficits [62].

(iii) The progressive volume loss of subcortical structures in patients revealed by scanning over two time points was not 
associated with pharmacotherapy, but rather to the underlying pathophysiology of this malignant form of schizophrenia illness and/or other illness-related factors, which were not present in controls. However, this explanation seems unlikely since patients in our cohort have a mean illness duration of 13 years and only some were in the early stages of illness.

The progressive loss of volume in subcortical structures despite symptomatic and functional improvement suggests that volume loss as detected by neuroimaging in vivo in our cohort should not be necessarily interpreted as harmful to patients. Although cognitive impairment has been related to cortical thinning or volume reduction in schizophrenia [63-65], grey matter loss has been associated with greater response to atypical antipsychotics $[66,67]$. Moreover, cortical thinning in first-episode schizophrenia patients on pharmacotherapy has been associated with physiological and cognitive improvement [68]. Consistent with this, progressive volumetric reduction of putamen and thalamus was significantly associated with better response to clozapine. This result was unaltered after controlling for the serum level of clozapine and duration of illness. Interestingly Scheepers et al., reported an association between clinical improvement in positive and general symptoms and reduction of left caudate volume, in TRS patients [20]. Molina et al., in a 2 years randomised clinical trial of clozapine on 17 neuroleptic-naïve patients with schizophrenia and 11 controls, have shown that inferior frontal thinning, specifically, pars orbitalis, opercularis and triangularis, was positively associated with better clinical and cognitive response to clozapine [69].

We also found that patients who were exposed to higher amounts of clozapine displayed a greater reduction of thalamus volume, this association was further reinforced when controlling for clinical symptoms and functioning, suggesting a direct effect of clozapine on the volumetric change of the thalamus. Vita et al. meta-analysis described a consistent finding where the greater the exposure to antipsychotics the greater the reduction in grey matter volume [15]. Two longitudinal studies have shown that the amount of exposure to antipsychotics predicted reduction of caudate and grey matter volumes [14] and the greater progressive brain reduction and ventricular enlargement were predicted by greater exposure to antipsychotic medication [70]. Although these studies have been interpreted as consistent with a toxic effect of antipsychotic medication on grey matter, generally patients were not randomised in these longitudinal studies and it is likely that patients with more severe illness were given larger amounts of medication. In our study other variables, such as age, duration of illness and daily dose of clozapine were not significant moderators of subcortical volume change over time, as previously reported [15].

A recent systematic review concludes that after 25 years of research it remains unclear, which are the biological predictors of symptomatic response to clozapine [71]. Greater integrity and activity in prefrontal cortical areas associated with a good response to clozapine is the most consistent finding, however, studies have failed to find any accurate and reproducible neuroanatomical biomarker to inform clinical decision-making. Although our study identified a relationship between thalamostriatal progression and clinical and functional improvement, we did not identify any baseline subcortical predictor of remission on clozapine.

\section{Strengths and limitations}

The main strength of this study is the longitudinal nature of a relatively large and homogenous sample of TRS patients. The careful segmentation of the subcortical structures using the longitudinal stream of Freesurfer based on an unbiased withinsubject anatomical template [35] enabled increased anatomical sensitivity to better detect anatomical changes and relationships to clinical symptoms and functioning. A potential limitation of this study is the lack of a comparative group of schizophrenia patients treated with other antipsychotic medications, in order to disentangle disease effects from treatment effects. However, such a comparative group may represent a less malign subgroup of patients with schizophrenia who are not treatment resistant and consequently may have a different underlying pathophysiology/ impact of antipsychotic medication on their neuroanatomy. Ultimately including MR imaging in longitudinal studies of schizophrenia where patients are randomised to different antipsychotic medications would be necessary to tease apart illness from treatment effects but only three such studies have been conducted to our knowledge $[60,69,72]$ and none on patients with treatment resistance. In addition, to reduce multiple analyses we assessed only subcortical structures summed bilaterally and did not explore any lateralised effects.

\section{CONCLUSION}

This study demonstrates that, despite the clinical and functional improvement of most patients with schizophrenia who are switched to clozapine, there is a counterintuitive progressive volume reduction in several subcortical structures over time. Furthermore, patients who have the greatest symptomatic improvement display the largest thalamo-striatal reductions, suggesting that volume reduction reflects an adaptive response associated with symptom improvement rather than a harmful process in these treatment-resistant patients. Further longitudinal studies with larger sample size, randomised designs and multimodal imaging will be necessary to disentangle the potentially dynamic effects of neuroprogression and antipsychotic treatment on different brain structures in schizophrenia.

\section{FUNDING AND DISCLOSURE}

This study was supported in part by the Wellcome Trust and the NUI Galway Millennium Project Fund and the Irish Research Council (GOIPG/2018/2464) Ph.D. Scholarship (GT). The authors declare no competing interests.

\section{ACKNOWLEDGEMENTS}

We would like to thank all the participants and their families for their involvement in the study.

\section{AUTHOR CONTRIBUTIONS}

Author CMCD designed and revised the paper for intellectual content; DMC and BH supervised the general progress of the study; MA recruited and collected data; LH collected data; TNA developed protocols for MRI processing. GT processed all the MRI data, performed statistical analyses and wrote the paper. All authors edited or approved the final paper.

\section{ADDITIONAL INFORMATION}

Supplementary Information accompanies this paper at (https://doi.org/10.1038/ s41386-020-0665-4).

Publisher's note Springer Nature remains neutral with regard to jurisdictional claims in published maps and institutional affiliations.

\section{REFERENCES}

1. Meltzer HY. Treatment-resistant schizophrenia-the role of clozapine. Curr Med Res Opin. 1997;14:1-20.

2. Lieberman JA, Safferman AZ, Pollack S, Szymanski S, Johns C, Howard A, et al. Clinical effects of clozapine in chronic schizophrenia: response to treatment and predictors of outcome. Am J Psychiatry. 1994;151:1744-52.

3. Suzuki T, Remington G, Mulsant BH, Uchida H, Rajji TK, Graff-guerrero A, et al. Defining treatment-resistant schizophrenia and response to antipsychotics: a review and recommendation. Psychiatry Res. 2012;197:1-6. 
4. Stroup TS, Mcevoy JP, Swartz MS, Byerly MJ, Qlick ID, Canive JM, et al. The National Institute of Mental Health Clinical Antipsychotic Trials of Intervention Effectiveness (CATIE) project: schizophrenia trial design and protocol development. Schizophr Bull. 2003;29:15-31.

5. Chakos M, Lieberman J, Hoffman E, Bradford D, Sheitman B. Effectiveness of second-generation antipsychotics in patients with treatment-resistant schizophrenia: a review and meta-analysis of randomized trials. Am J Psychiatry. 2001;158:518-26.

6. Henderson DC, Cagliero E, Hayden DL, Schoenfeld DA, Goff DC. Clozapine, Diabetes Mellitus, Weight Gain, and Lipid Abnormalities: A Five-Year Naturalistic Study. Am J Psychiatry. 2000;157:975-81.

7. Meltzer HY, Perry E, Jayathilake K. Clozapine-induced weight gain predicts improvement in psychopathology. Schizophr Res. 2003;59:19-27.

8. Lally J, Gallagher A, Bainbridge E, Avalos G, Ahmed M, Mcdonald C. Increases in triglyceride levels are associated with clinical response to clozapine treatment. J Psychopharmacol. 2013. https://doi.org/10.1177/0269881112472568.

9. Anderson VM, Goldstein ME, Kydd RR, Russell BR. Extensive gray matter volume reduction in treatment-resistant schizophrenia. Int J Neuropsychopharmacol. 2015;18:1-10.

10. Molina V, Reig S, Sanz J, Palomo T, Benito C, Sarramea F, et al. Differential clinical, structural and $\mathrm{P} 300$ parameters in schizophrenia patients resistant to conventional neuroleptics. Prog Neuropsychopharmacol Biol Psychiatry. 2008;32:257-66.

11. Kubera KM, Sambataro F, Vasic N, Wolf ND, Frasch K, Hirjak D, et al. Source-based morphometry of gray matter volume in patients with schizophrenia who have persistent auditory verbal hallucinations. Prog Neuropsychopharmacol Biol Psychiatry. 2014:50:102-9.

12. Quarantelli M, Palladino O, Prinster A, Schiavone V, Carotenuto B, Brunetti A, et al. Patients with poor response to antipsychotics have a more severe pattern of frontal atrophy: a voxel-based morphometry study of treatment resistance in schizophrenia. Biomed Res Int. 2014;2014:325052.

13. Zugman A, Gadelha A, Assuncao I, Sato J, Ota VK, Rocha DL, et al. Reduced dorsolateral prefrontal cortex in treatment resistant schizophrenia. Schizophr Res. 2013;148:81-6.

14. Ho B-C, Andreasen N, Ziebel S, Pierson R, Magnotta V. Long-term antipsychotic treatment and brain volumes: a longitudinal study of first-episode schizophrenia. Arch Gen Psychiatry. 2011;68:128-37.

15. Vita A, Peri De L, Deste G, Barlati S, Sacchetti E. Archival report the effect of antipsychotic treatment on cortical gray matter changes in schizophrenia: does the class matter? a meta-analysis and meta-regression of longitudinal magnetic resonance imaging studies. Biol Psychiatry. 2015;78:403-12.

16. van Haren NEM, Schnack HG, Cahn W, Van Den Heuvel MP, Lepage C, Collins L, et al. Changes in cortical thickness during the course of illness in schizophrenia. Arch Gen Psychiatry. 2011;68:871-80.

17. van Haren NEM, Hulshoff Pol HE, Schnack HG, Cahn W, Mandl RCW, Collins DL, et al. Focal gray matter changes in schizophrenia across the course of the illness: a 5-year follow-up study. Neuropsychopharmacology. 2007:32:2057-66.

18. Chakos MH, Lieberman JA, Alvir J, Bilder R, Ashtari M. Caudate nuclei volumes in schizophrenic patients treated with typical antipsychotics or clozapine. Lancet. 1995;345:456-7.

19. Scheepers FE, Pol HEH, van der Flier W, Hijman R, van der Linden JA, de Wied CCG, et al. The effect of clozapine on the nucleus caudate volume in schizophrenic patients previously treated with classical neuroleptics. Schizophr Res. 2001;36:208.

20. Scheepers FE, Gispen de Wied CC, Hulshoff Pol HE, Kahn RS. Effect of clozapine on caudate nucleus volume in relation to symptoms of schizophrenia. Am J Psychiatry. 2001;158:644-6.

21. Frazier J, Giedd JN, Kaysen D, Rad JA. Childhood-onset schizophrenia: brain MRI rescan after 2 years of clozapine maintenance treatment. 1996. https://doi.org/ 10.1176/ajp.153.4.564

22. Seeman $P$, Wilson $A$, Gmeiner $P$, Kapur S. Dopamine D2 and D3 receptors in human putamen, caudate nucleus, and globus pallidus. Synapse. 2006;60:205-11.

23. Arango C, Buchanan RW, Breier A, MacMahon R, Carpenter WT. The relationship of clozapine and haloperidol treatment response to prefrontal, hippocampal, and caudate brain volumes. Am J Psychiatry. 2003;160:1421-7.

24. Molina V, Reig S, Sarramea F, Sanz J, Artaloytia JF, Luque R, et al. Anatomical and functional brain variables associated with clozapine response in treatmentresistant schizophrenia. Psychiatry Res: Neuroimaging. 2003;124:153-61.

25. Ahmed M, Cannon DM, Scanlon C, Holleran L, Schmidt H, McFarland J, et al. Progressive brain atrophy and cortical thinning in schizophrenia after commencing clozapine treatment. Neuropsychopharmacology. 2015;40:2409-17.

26. National Institute of Health and Clinical Excellence. Psychosis and schizophrenia in adults. NICE Guidelines Treament Management. National Collaborating Centre For Mental Health; 2014. p. 74-80.

27. Kay SR, Fiszbein A, Opler LA. The positive and negative syndrome scale (PANSS) for schizophrenia. Schizophr Bull. 1987;13:261-76.
28. Andreasen NC. The Scale for the Assessment of Positive Symptoms (SAPS). Lowa City: University of Lowa; 1982.

29. Andreasen NC. The Scale for the Assessment of Negative Symptoms (SANS). Lowa City: University of Lowa; 1982.

30. Hall RCW. Global assessment of functioning: a modified scale. Psychosomatics. 1995:36:267-75

31. Andreasen NC, Carpenter WT, Kane JM, Lasser RA, Marder SR, Weinberger DR. Remission in schizophrenia: proposed criteria and rationale for consensus. Am J Psychiatry. 2005;162:441-9.

32. Egerton A, Broberg BV, Van Haren N, Merritt K, Barker GJ, Lythgoe DJ, et al. Response to initial antipsychotic treatment in first episode psychosis is related to anterior cingulate glutamate levels: a multicentre $1 \mathrm{H}$-MRS study (OPTiMiSE). Mol Psychiatry. 2018;23:2145-55.

33. Sled JG, Zijdenbos AP, Evans AC. A nonparametric method for automatic correction of intensity nonuniformity in MRI data. IEEE Trans Med Imaging. 1998;17:87-97.

34. Scanlon C, Anderson-Schmidt H, Kilmartin L, Mclnerney S, Kenney J, McFarland J, et al. Cortical thinning and caudate abnormalities in first episode psychosis and their association with clinical outcome. Schizophr Res. 2014;159:36-42.

35. Reuter M, Schmansky NJ, Rosas HD, Fischl B. Within-subject template estimation for unbiased longitudinal image analysis. Neuroimage. 2012;61:1402-18.

36. FreeSurfer. 2013. https://surfer.nmr.mgh.harvard.edu/. Accessed 2 Oct 2018.

37. Reuter M, Rosas HD, Fischl B. Highly accurate inverse consistent registration: a robust approach. Neuroimage. 2010;53:1181-96.

38. Tronchin G, Akudjedu TN, Kenney J, Mclnerney S, Scanlon C, McFarland J, et al. Association between executive functions at first episode of psychosis and the later development of prefrontal cortical thinning and negative symptoms. European Neuropsychopharmacology. 2019;29:S412-S413.

39. eTIV. http://surfer.nmr.mgh.harvard.edu/fswiki/eTIV. 2019.

40. talairach_avi. https://surfer.nmr.mgh.harvard.edu/fswiki/talairach_avi. 2019.

41. Evans AC, Collins DL, Mills SR, Brown ED, Kelly RL, Peters TM. 3D statistical neuroanatomical models from $305 \mathrm{MRI}$ volumes. In: IEEE conference record nuclear science symposium and medical imaging conference; 1993. p. 1813-7.

42. FreeSurfer Quality Control Guide. 2013. https://sites.bu.edu/cnrlab/lab-resources/ freesurfer-quality-control-guide/. Accessed 2 Oct 2018

43. ControlPoints_freeview. https://surfer.nmr.mgh.harvard.edu/fswiki/FsTutorial/ ControlPoints freeview. 2018.

44. Van Erp TGM, Hibar DP, Rasmussen JM, Glahn DC, Pearlson GD, Andreassen OA, et al. Subcortical brain volume abnormalities in 2028 individuals with schizophrenia and 2540 healthy controls via the ENIGMA consortium. Mol Psychiatry. 2016;21:547-53.

45. Westmoreland Corson P, Nopoulos P, Miller DD, Arndt S, Andreasen NC. Change in basal ganglia volume over 2 years in patients with schizophrenia: typical versus atypical neuroleptics. Am J Psychiatry. 1999;156:1200-4.

46. Lang DJ, Kopala LC, Vandorpe RA, Rui Q, Smith GN. et al. Reduced basal ganglia volumes after switching to olanzapine in chronically treated patients with schizophrenia. Am J Psychiatry. 2004;161:1829-36.

47. Crespo-facorro B, Roiz-santiáñez R, Pérez-iglesias R, Pelayo-terán JM, Rodríguezsánchez JM, Tordesillas-gutiérrez $D$, et al. Progress in neuropsychopharmacology \& biological psychiatry effect of antipsychotic drugs on brain morphometry. a randomized controlled one-year follow-up study of haloperidol, risperidone and olanzapine. Prog Neuropsychopharmacol Biol Psychiatry. 2008:32:1936-43.

48. Ho NF, Iglesias JE, Sum MY, Kuswanto CN, Sitoh YY, De Souza J, et al. Progression from selective to general involvement of hippocampal subfields in schizophrenia. Mol Psychiatry. 2017;22:142-52.

49. Kempton MJ, Stahl D, Williams SCR, DeLisi LE. Progressive lateral ventricular enlargement in schizophrenia: a meta-analysis of longitudinal MRI studies. Schizophr Res. 2010;120:54-62.

50. Howes OD, Kapur S. The dopamine hypothesis of schizophrenia: version III-the final common pathway. Schizophr Bull. 2009;35:549-62.

51. Money KM, Stanwood GD. Developmental origins of brain disorders: roles for dopamine receptor expression patterns. Front Cell Neurosci. 2013;7:1-14.

52. Guma E, Rocchetti J, Devenyi GA, Tanti A, Mathieu A, Lerch JP, et al. Neurolmage regional brain volume changes following chronic antipsychotic administration are mediated by the dopamine D2 receptor. Neuroimage. 2018;176:226-38.

53. Lally J, Gaughran F, Timms P, Curran SR. Treatment-resistant schizophrenia: current insights on the pharmacogenomics of antipsychotics. Pharmgenom Pers Med. 2016;9:117-29.

54. Gillespie AL, Samanaite R, Mill J, Egerton A, Maccabe JH. Is treatment-resistant schizophrenia categorically distinct from treatment-responsive schizophrenia? a systematic review. BMC Psychiatry. 2017;17:1-14.

55. Howes OD, Kapur S. A neurobiological hypothesis for the classification of schizophrenia: type a (hyperdopaminergic) and type b (normodopaminergic). Br J Psychiatry. 2014;205:1-3. 
56. Goldstein ME, Anderson VM, Pillai A, Kydd RR, Russell BR. Glutamatergic neurometabolites in clozapine-responsive and -resistant schizophrenia. Int J Neuropsychopharmacol. 2015;18:1-9.

57. Amitai N, Kuczenski R, Behrens MM, Markou A. Neuropharmacology repeated phencyclidine administration alters glutamate release and decreases GABA markers in the prefrontal cortex of rats. Neuropharmacology. 2012;62:1422-31.

58. Graeber MB, Li W, Rodriguez ML. Role of microglia in CNS inflammation. FEBS Lett. 2011;585:3798-805.

59. Al-amin M, Muhammad M, Uddin N, Reza HM. Effects of antipsychotics on the inflammatory response system of patients with schizophrenia in peripheral blood mononuclear cell cultures. Clin. Psychopharmacol. Neurosci. 2013;11:144-51.

60. Lieberman JA, Tollefson GD, Charles C, Zipursky R, Sharma T, Kahn RS, et al. Antipsychotic drug effects on brain morphology in first-episode psychosis. Arch Gen Psychiatry. 2005;62:361-70.

61. Haijma SV, Van Haren N, Cahn W, PCMP Koolschijn, Hulshoff Pol HE, Kahn RS Brain volumes in schizophrenia: a meta-analysis in over 18000 subjects. Schizophr Bull. 2013;39:1129-38.

62. Ho B-C, Andreasen NC, Nopoulos P, Arndt S, Magnotta V, Flaum M. Progressive structural brain abnormalities and their relationship to clinical outcome: a longitudinal magnetic resonance imaging study early in schizophrenia. Arch Gen Psychiatry. 2003;60:585-94.

63. Antonova E, Kumari V, Morris R, Halari R, Anilkumar A, Mehrotra R, et al. The relationship of structural alterations to cognitive deficits in schizophrenia: a voxel-based morphometry study. Biol Psychiatry. 2005;58:457-67.

64. Fan F, Xiang H, Tan S, Yang F, Fan H, Guo H, et al. Subcortical structures and cognitive dysfunction in first episode schizophrenia. Psychiatry Res Neuroimaging. 2019;286:69-75
65. Koshiyama D, Fukunaga M, Okada N, Yamashita F, Yamamori H, Yasuda $Y$, et al Role of subcortical structures on cognitive and social function in schizophrenia. Sci Rep. 2018;8:1183.

66. Sporn AL, Greenstein DK, Gogtay N, Jeffries NO, Lenane M, Gochman P, et al. Progressive brain volume loss during adolescence in childhood-onset schizophrenia. Am J Psychiatry. 2003;160:2181-9.

67. Gur RE, Cowell P, Turetsky BI, Gallacher F, Cannon T, Bilker W, et al. A follow-up magnetic resonance imaging study of schizophrenia. Arch Gen Psychiatry. 1998;55:145.

68. Lesh TA, Tanase C, Geib BR, Niendam TA, Yoon JH. A multimodal analysis of antipsychotic effects on brain structure and function a multimodal analysis of antipsychotic effects on brain structure and function in first-episode schizophrenia. 2015. https://doi.org/10.1001/jamapsychiatry.2014.2178.

69. Molina V, Taboada D, Aragüés M, Hernández JA, Sanz-Fuentenebro J. Greater clinical and cognitive improvement with clozapine and risperidone associated with a thinner cortex at baseline in first-episode schizophrenia. Schizophr Res. 2014;158:223-9.

70. Guo JY, Huhtaniska S, Miettunen J, Jääskeläinen E, Kiviniemi V, Nikkinen J, et al Longitudinal regional brain volume loss in schizophrenia: relationship to antipsychotic medication and change in social function. Schizophr Res. 2015;168:297-304.

71. Samanaite R, Gillespie A, Sendt K, Mcqueen G. Biological predictors of clozapine response: a systematic review. Front Psychiatry. 2018;9:1-29.

72. Roiz-santiáñez R, Tordesillas-gutiérrez $D$, De VO, Ayesa-arriola R, Gutiérrez A, Tabarés-seisdedos $R$, et al. Effect of antipsychotic drugs on cortical thickness. a randomized controlled one-year follow-up study of haloperidol, risperidone and olanzapine. Schizophr Res. 2012;141:22-8. 